\title{
Coherent backscattering of light
}

\author{
R. Corey, M. Kissner, and P. Saulnier \\ Department of Physics, Gustavus Adolphus College \\ Saint Peter, MN 56082
}

\begin{abstract}
Coherent backscattering of light (CBS) is a phenomenon in which partial waves traversing time-reversed (momentum-reversed) scattering paths interfere constructively in the backscattering direction leading to the appearance of an intensity cone. This increase in reflectivity reduces the amount of light transported through the colloidal media. As an advanced undergraduate laboratory CBS introduces the student to low-level light detection, precision angular resolved measurements, and sophisticated data analysis techniques. Additionally, the concept of photon self-interference, usually discussed in Modern Physics class in the context of Young's double slit experiment, is beautifully illustrated by CBS.
\end{abstract}

PACS numbers: 42.25.Bs, 01.50.Pa, 42.68.Ay, 42.68.Mj 


\section{INTRODUCTION}

Light scattering experiments have a long and time honored history in physics. Early experimental investigations into the nature and behavior of light were conducted by Newton, Huygens, Young, Fresnel, and Rayleigh to name a few. Our understanding has progressed from a belief in ether borne vibrations to a self-consistent electromagnetic theory that incorporates all known light scattering phenomena. Light scattering experiments have enjoyed a renaissance since the invention of the laser and have gained further prominence as potential applications of quantum optics, nonlinear optical materials, and photon localization are conceived. It is the possibility of achieving photon localization [1] that has spurred interest in coherent backscattering of light (CBS) as a current research topic. This experiment also has much to offer as an advanced undergraduate laboratory.

Coherent backscattering of light is a photon self-interference effect which leads to an enhanced intensity cone in the backscattering direction. A schematic representation of the now classic CBS experiment is illustrated in Fig. 1. Laser light is incident on a dense colloidal suspension containing polystyrene spheres [2], in water, undergoing Brownian motion. The diffuse intensity profile is recorded as a function of backscattering angle leading to the appearance of the CBS intensity cone, whose height is predicted to be twice the incoherent background level. This effective increase in reflectivity reduces the optical energy transported in the forward direction and provides the link with photon localization. This retro reflection enhancement was first observed by A. Ishimaru and Y. Kuga [3] but it was not until the observations of M. van Albada and A. Lagendijk [4] and G. Maret and P. Wolf [5] that its connection with phase coherent time-reversed paths and photon localization was made clear. This connection is sometimes made explicit by referring to CBS as weak photon localization. 


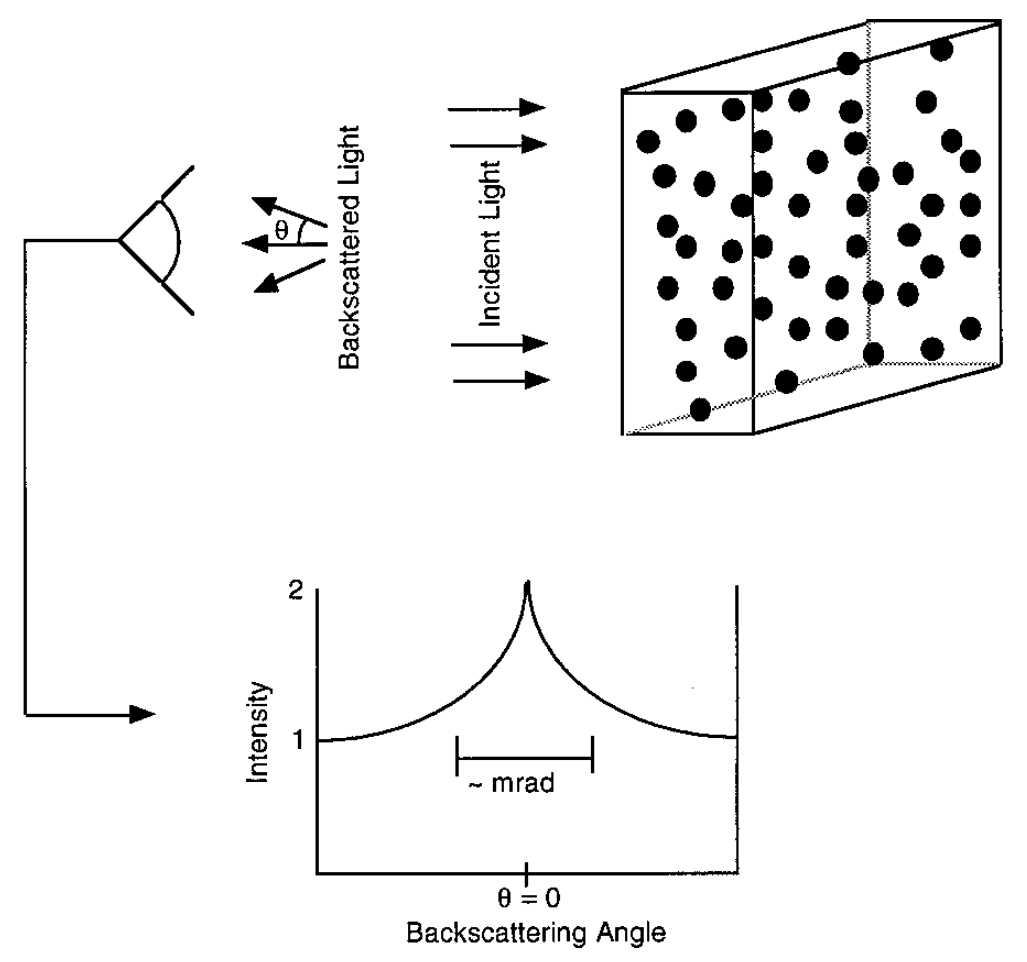

Figure 1: A schematic representation of a coherent backscattering experiment. Backscattered intensity from a colloidal suspension is recorded as a function of backscattering angle $\theta$. The characteristic cusp shaped intensity profile with the theoretically predicted cone enhancement of two is illustrated.

In order to understand the origin of this enhanced intensity cone, the nature of the photon scattering within the colloidal suspension must be examined. The simplest model describing this process is that the photons are undergoing a diffusion-like random-walk with an average step size given by the transport mean-free-path length $\ell^{*}$. However, since light in this experiment behaves as an electromagnetic wave, and not a classical particle, interference between scattering paths must be considered. It is just this interference between time-reversed (momentum-reversed) paths that gives rise to the CBS intensity cone. Figure 2 shows a prototypical scattering path and its time-reversed mate and also illustrates the existence of a path length difference (PLD) between the two. 


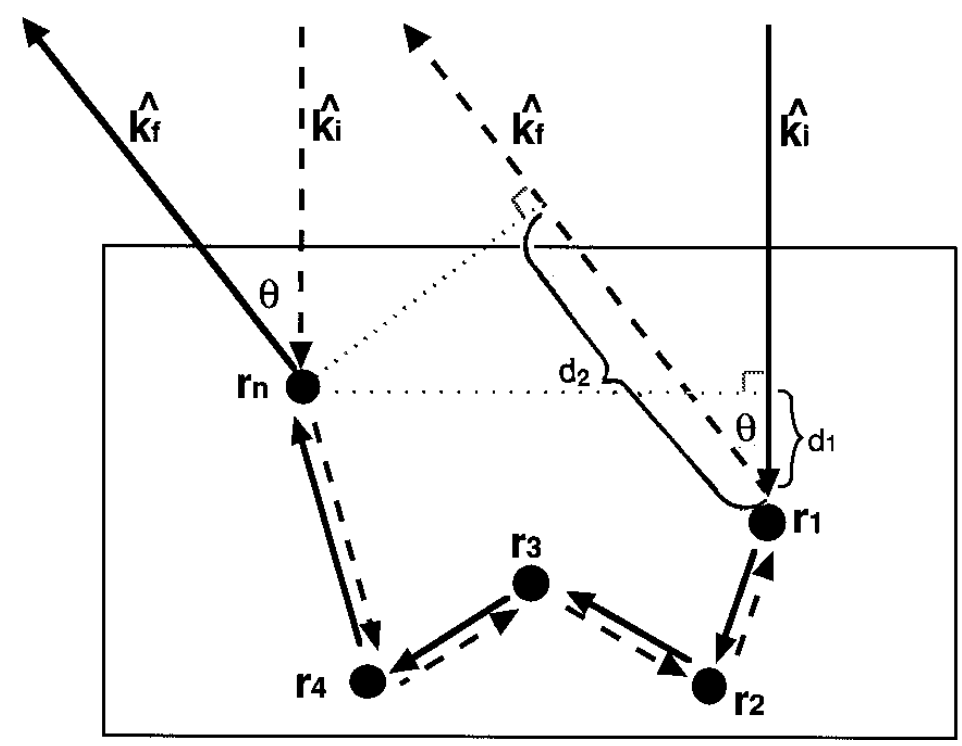

Figure 2: A prototypical scattering path (solid line) and its time-reversed mate (dashed line) with incident light direction $\widehat{\mathbf{k}}_{\mathrm{i}}$ and final direction $\widehat{\mathbf{k}}_{\mathrm{f}}$. Five scattering centers are shown with positions given by $\mathbf{r}_{1}$ through $\mathbf{r}_{\mathrm{n}}$, relative to an arbitrary origin. The last scattering center is labeled $\mathbf{r}_{\mathrm{n}}$ since the results of the analysis hold for any scattering order beyond single scattering. The intermediate scattering vectors, $\widehat{\mathbf{k}}$ 's, are not labeled for clarity. The optical path length difference between these two time-reversed scattering paths only depends on the physical path length difference $d_{2}-d_{1}$. The scattering angle $\theta$, relative to the backscattered direction $-\widehat{\mathbf{k}}_{\mathrm{i}}$, is also shown. When light is directly backscattered, $\widehat{\mathbf{k}}_{\mathrm{f}}=-\widehat{\mathbf{k}}_{\mathrm{i}}, \theta$ is zero and the optical path length difference vanishes since $d_{2}=d_{1}$, leading to constructive interference.

The nature of the time-reversed scattering path interference depends on the phase difference between the two scattered waves. This phase difference is given as

$$
\Delta \phi=\frac{2 \pi}{\lambda} \mathrm{PLD}=\frac{2 \pi}{\lambda}\left(d_{2}-d_{1}\right)
$$

where $\lambda$ is the wavelength of the incident radiation in water, PLD is again the path length difference, the distance $d_{1}$ (indicated in Fig. 2) is the projection of the vector that points from $\mathbf{r}_{1}$ to $\mathbf{r}_{\mathrm{n}}$, that is $\left(\mathbf{r}_{\mathrm{n}}-\mathbf{r}_{1}\right.$ ), on to $-\widehat{\mathbf{k}}_{\mathrm{i}}$, and the distance $d_{2}$ (also indicated in Fig. 2) is the projection of vector $\left(\mathbf{r}_{\mathrm{n}}-\mathbf{r}_{1}\right)$ on to $\widehat{\mathbf{k}}_{\mathrm{f}}$. The distances $d_{1}$ and $d_{2}$ are thus given by

$$
\begin{aligned}
d_{1} & =-\widehat{\mathbf{k}}_{\mathrm{i}} \cdot\left(\mathbf{r}_{\mathrm{n}}-\mathbf{r}_{1}\right) \\
d_{2} & =\widehat{\mathbf{k}}_{\mathrm{f}} \cdot\left(\mathbf{r}_{\mathrm{n}}-\mathbf{r}_{1}\right)
\end{aligned}
$$

which yields a phase difference of 


$$
\Delta \phi=\frac{2 \pi}{\lambda}\left(\widehat{\mathbf{k}}_{\mathrm{f}}+\widehat{\mathbf{k}}_{\mathrm{i}}\right) \cdot\left(\mathbf{r}_{\mathrm{n}}-\mathbf{r}_{1}\right)
$$

It is evident from this expression that for light scattered directly into the backward direction, $\widehat{\mathbf{k}}_{\mathrm{f}}=-\widehat{\mathbf{k}}_{\mathrm{i}}$, the phase difference between a scattering path and its timereversed mate is zero, and thus, completely constructive interference results.

The qualitative nature of the interference away from the exact backscattering direction can be ascertained by making use of our diffusive model of photon transport within the colloidal suspension. The phase difference becomes, by evaluating the dot product in Eq.(1.3),

$$
\Delta \phi=\frac{2 \pi}{\lambda} 2 \sin (\theta / 2) R \cos \alpha
$$

where $\theta$ is the scattering angle measured from the backscattering direction, $R$ is $\left|\mathbf{r}_{\mathrm{n}}-\mathbf{r}_{1}\right|$, $\alpha$ is the angle between $\left(\widehat{\mathbf{k}}_{\mathrm{f}}+\widehat{\mathbf{k}}_{\mathrm{i}}\right)$ and $\left(\mathbf{r}_{\mathrm{n}}-\mathbf{r}_{1}\right)$, and where we have used $\left|\widehat{\mathbf{k}}_{\mathrm{f}}+\widehat{\mathbf{k}}_{\mathrm{i}}\right|=2 \sin (\theta / 2)$. Restricting our analysis to the experimentally realized situation of small $\theta$ (milliradians) we obtain

$$
\Delta \phi \approx \frac{2 \pi}{\lambda} \theta R
$$

where we have also used the fact that for a dense colloidal suspension $\left(\mathbf{r}_{\mathrm{n}}-\mathbf{r}_{1}\right)$ will be nearly parallel to the sample surface and $\left(\widehat{\mathbf{k}}_{\mathrm{f}}+\widehat{\mathbf{k}}_{\mathrm{i}}\right)$, yielding $\cos \alpha \approx 1$. The meansquare separation between the first and last scatterer in the photon diffusion approximation is given as

$$
\left\langle R^{2}\right\rangle=6 D t=6\left(\frac{c \ell^{*}}{3}\right) t
$$

where $D$ is the photon diffusion coefficient in the colloidal suspension, $t$ is the randomwalk time, and $c$ is the velocity of light propagation [6]. Using the root-mean-square value for $R$ we obtain the final expression for the phase difference

$$
\Delta \phi \approx \frac{2 \pi}{\lambda} \theta \sqrt{6\left(\frac{c \ell^{*}}{3}\right) t}=\frac{2 \pi}{\lambda} \theta \sqrt{2 \ell^{*} s}
$$

where $s=c t$, the total scattering path length.

In order for the two partial waves which traverse time-reversed paths to add constructively, their phase difference must be small. This coherence condition may be stated as $\frac{\Delta \phi}{2 \pi} \ll 1$. Thus, there will exist a critical angle $\theta_{\mathrm{c}}$ below which this condition 
will be satisfied and phase coherence maintained. This critical angle is

$$
\theta_{\mathrm{c}} \approx \frac{\lambda}{\sqrt{2 \ell^{*} s}}
$$

This result has important implications for the shape of the coherent backscattering cone and accounts for the cone enhancement factor, the ratio of maximum cone height to background level, of two. As mentioned above, Eq.(1.3) implies that the two partial waves must be added coherently (sum then square) to obtain their contribution to the intensity in the exact backscattering direction, whereas the incoherent sum (square then add) may be used for angles greater than $\theta_{\mathrm{c}}$, leading to the enhancement factor of two.

One implication for the cone shape is that paths for which the total path length $s$ is large will only contribute coherently within a relatively small angle about the backscattering direction. That is, the critical angle decreases as the total photon path length increases. This implies that any physical phenomenon that affects the long scattering paths, or distribution of path lengths, will manifest itself near the backscattering direction. Some of these phenomena include absorption [7], finite sample size [8], and sample surface reflectivity [9].

Another important result that is easily obtained from Eq.(1.8) is the dependence of the coherent cone width on the transport mean-free-path length. The maximum angular cone width is the critical coherence angle obtained for the smallest total path length. The smallest average path length is $s=\ell^{*}$, yielding a cone width of

$$
\theta_{\max } \approx \frac{\lambda}{\sqrt{2} \ell^{*}}
$$

which scales inversely with $\ell^{*}$.

An analytic expression for the coherent backscattering intensity line shape may be obtained within the diffusion approximation [7] and is given by

$$
I(q)=\frac{1}{2} \frac{3}{8 \pi}\left[1+2 \frac{z_{0}}{\ell^{*}}+\frac{1}{\left(1+q \ell^{*}\right)^{2}}\left(1+\frac{1-e^{-2 q z_{0}}}{q \ell^{*}}\right)\right]
$$

where $q=2 \pi \theta / \lambda$ and $z_{0} \approx \frac{2}{3} \ell^{*}$. Among the assumptions contained in the diffusion approximation is that of scalar waves, which have no polarization state by definition. In a coherent backscattering experiment one may analyze and detect the incident polarization or its complement. The choice of polarization state detected profoundly influences the observed intensity lineshape. Equation (1.10) is valid if the detected polarization is identical to the incident polarization, the so-called helicity preserving channel. 


\section{EXPERIMENT AND RESULTS}

The experimental configuration used to measure coherent backscattering intensity profiles is shown in Fig. 3. A low power $(\approx 20 \mathrm{~mW})$, air-cooled Argon-ion laser (488 $\mathrm{nm})$ was used as the light source, although a less expensive HeNe laser $(\approx 10 \mathrm{~mW})$ will also perform well. The laser beam was expanded to reduce beam divergence and thus the uncertainty in the incident photon direction $\widehat{\mathbf{k}}_{\mathrm{i}}$. A modified Michelson interferometer arrangement was employed to allow detection of the backscattered intensity. The plate beam splitter used in the experiment was anti-reflection coated to eliminate unwanted false images. A quarter-wave plate and an analyzer were used as an "optical diode" to detect only photons in the helicity preserving channel. The quarter-wave plate converted the incident vertical polarization to right circularly polarized light which was then incident upon the colloidal suspension. Backscattered light that is right circularly polarized will be converted back to vertical polarization upon traversing the quarter-wave plate for the second time, whereas left circularly polarized light will be converted to horizontal polarization which will be blocked by the vertical transmission axis of the analyzer. Besides allowing for the detection of the helicity preserving channel this optical arrangement has the added benefit of suppressing the single scattering contribution to the backscattered intensity. This suppression is a result of a polarization helicity flip upon single scattering occurring due to the necessity of maintaining nodality at the scattering interface (a $\pi$ phase shift). The suppression of single scattering is important since single scattering contributes to the incoherent background level but not the coherent peak, owing to the lack of a time-reversed mate, and thus reduces the observed enhancement factor. The light that passed through the analyzer was focused by a $700 \mathrm{~mm}$ focal length lens into a $200 \mu \mathrm{m}$ pinhole through a $488 \mathrm{~nm}$ bandpass filter to the photomultiplier tube (PMT). This geometrical configuration resulted in an experimental angular resolution of approximately 0.3 milliradians which is sufficient to resolve coherent cones from all but the most dilute colloidal suspensions. 


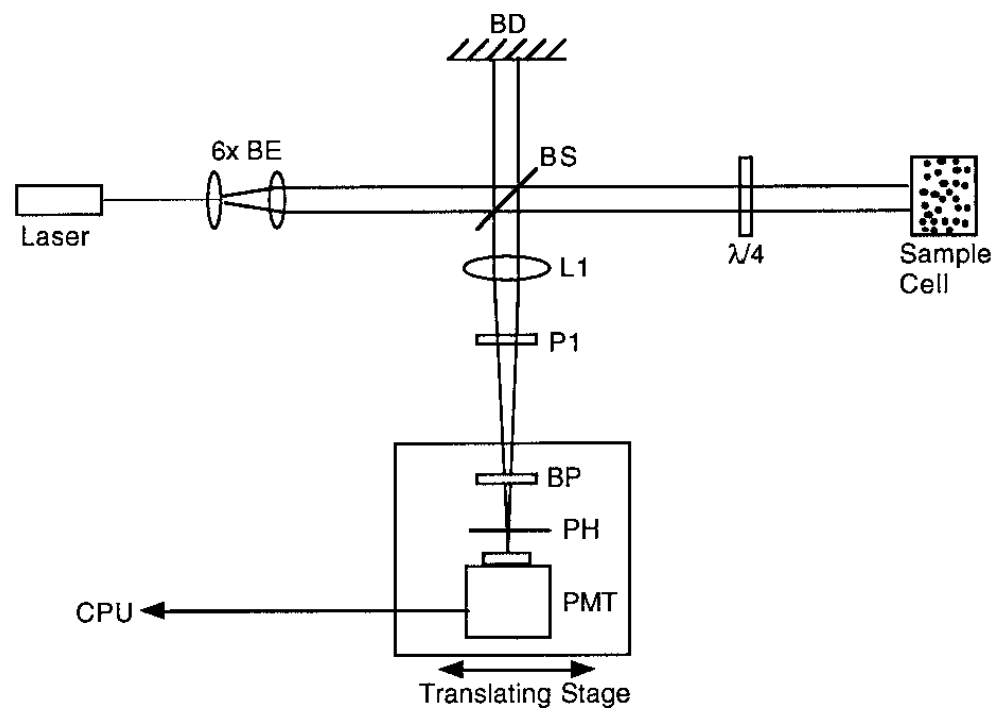

Figure 3: The experimental configuration used to measure coherent backscattering intensity profiles. An Argon-ion laser $(488 \mathrm{~nm})$ was used along with other major components; BE, beam expander; BS, beam splitter; BD, beam dump; $\lambda / 4$, quarter-wave plate; L1, $700 \mathrm{~mm}$ focal length lens; P1, analyzer; BP, $488 \mathrm{~nm}$ bandpass filter; and PH, $200 \mu \mathrm{m}$ pinhole.

The photomultiplier tube assembly used was Hamamatsu Corporation HC120-05. This air-cooled PMT is ideal for use in student laboratories due to its small size $(19 \mathrm{~mm} \mathrm{x}$ $51 \mathrm{~mm} \times 53 \mathrm{~mm}$ ) and the necessity of only supplying it with \pm 15 volts. Its small size and lack of water cooling requirements make it ideally suited for translation, while its onboard high voltage generation adds to its safety. The photomultiplier tube bias voltage was set to maximize signal-to-noise ratio. This was accomplished by plotting count rate at constant light input versus bias voltage and operating the PMT at a voltage near the beginning of the plateau exhibited in this curve. The PMT output was sent through a preamp and into a Stanford Research SR400 photon counter. The photon counter's internal discriminator was set to minimize the dark count, which was approximately 20 counts per second. The discriminator and bandpass filter were sufficient to adequately reduce the background light. The integration time was chosen to provide maximum signal strength while maintaining a reasonable total scan time. The total scan time $\approx 5$ minutes was chosen so that no sample variation occurred during this time. Students acquired direct experience with the tradeoff between integration time and signal-to-noise ratio. A lower cost alternative to the research grade SR400 $(\approx \$ 5,000)$ would be to pass the PMT output through a pulse stretching and conditioning circuit and into a standard countercard $(\approx \$ 500)$ in a computer. The actual experimental scan was automated and 
conducted under computer control. The computer moved the pinhole/photomultiplier tube assembly in approximately $0.05 \mathrm{~mm}$ increments and instructed the photon counter to begin integrating. Upon completion of the counting cycle the data would be transferred to the computer via a GPIB interface.

A typical coherent backscattering intensity profile, obtained from a $10 \% 0.966 \mu \mathrm{m}$ polystyrene colloidal suspension, and the corresponding theoretical fit is shown in Fig. 4. The theoretical model given in Eq.(1.10) was fit to the data using a modified MarquardtLevenberg nonlinear least squares fitting algorithm [10]. The theoretical model was convolved with the system response function prior to its comparison with the data in order to account for the finite experimental angular resolution. The system response function was obtained by replacing the sample with a mirror, directed in the backscattering direction, and conducting a normal scan. The theoretically predicted enhancement factor of two was experimentally difficult to realize. This difficulty is due to several factors including finite sample cell size (eliminating the possibility of very long scattering paths), finite angular resolution, failure to completely eliminate single scattering, and the presence of closed loop scattering paths. Closed loop scattering paths contribute coherently for any scattering direction $\theta$ since for such paths $\mathbf{r}_{\mathrm{n}}=\mathbf{r}_{1}$ implying $\Delta \phi=0$ independent of $\widehat{\mathbf{k}}_{\mathrm{f}}$. This closed loop contribution to the backscattered intensity profile is a topic of current research.

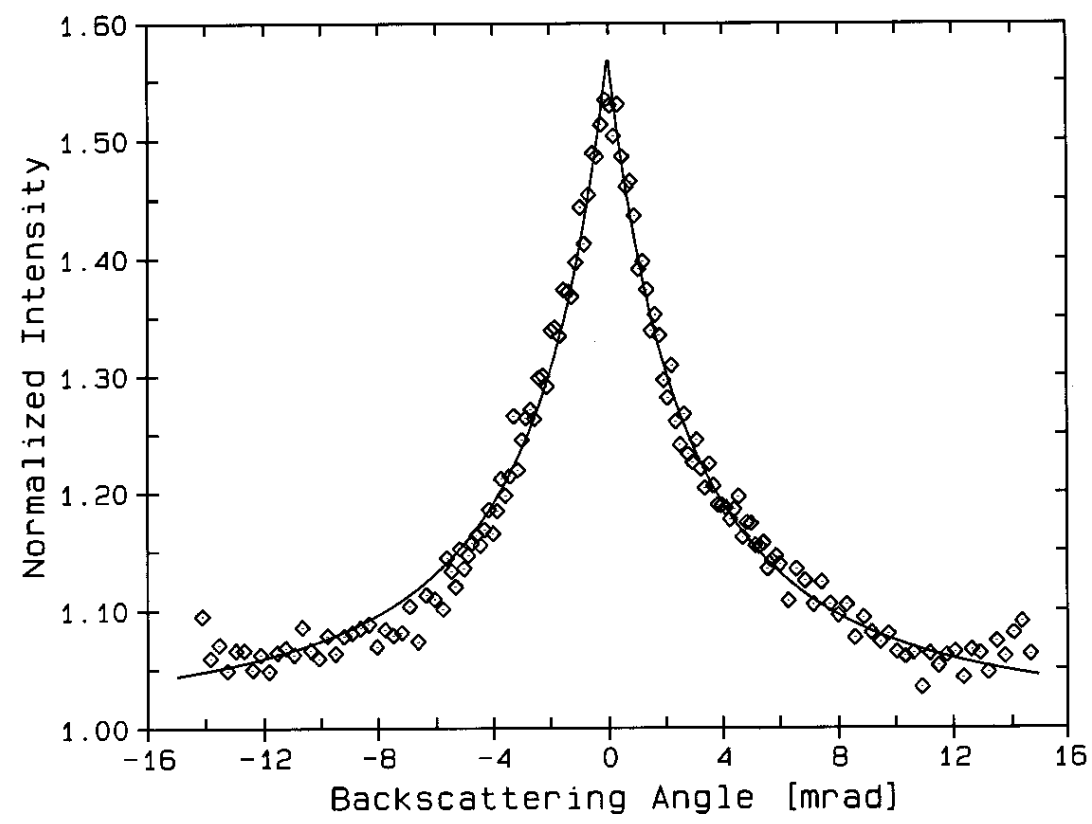

Figure 4: A typical coherent backscattering intensity profile with the corresponding theoretical model which was convolved with the system response function. This data was obtained using a $10 \%$ colloidal suspension of $0.966 \mu \mathrm{m}$ polystyrene spheres in water. 
The width of coherent backscattering cones has been shown to be inversely proportional to the transport mean-free-path length $\ell^{*}$, see Eq.(1.9). During the course of our investigation this dependence was experimentally verified by recording the coherent intensity profiles for various concentrations of a colloidal suspension. The width of these coherent cones was plotted versus $k \ell^{*}$ where $k=2 \pi / \lambda$ and $\ell^{*}$ was obtained from Mie theory [11] appropriately corrected for particle-particle correlations [12]. The result of this analysis is plotted in Fig. 5 and as expected the inverse dependence on $\ell^{*}$ is observed on this log-log plot.

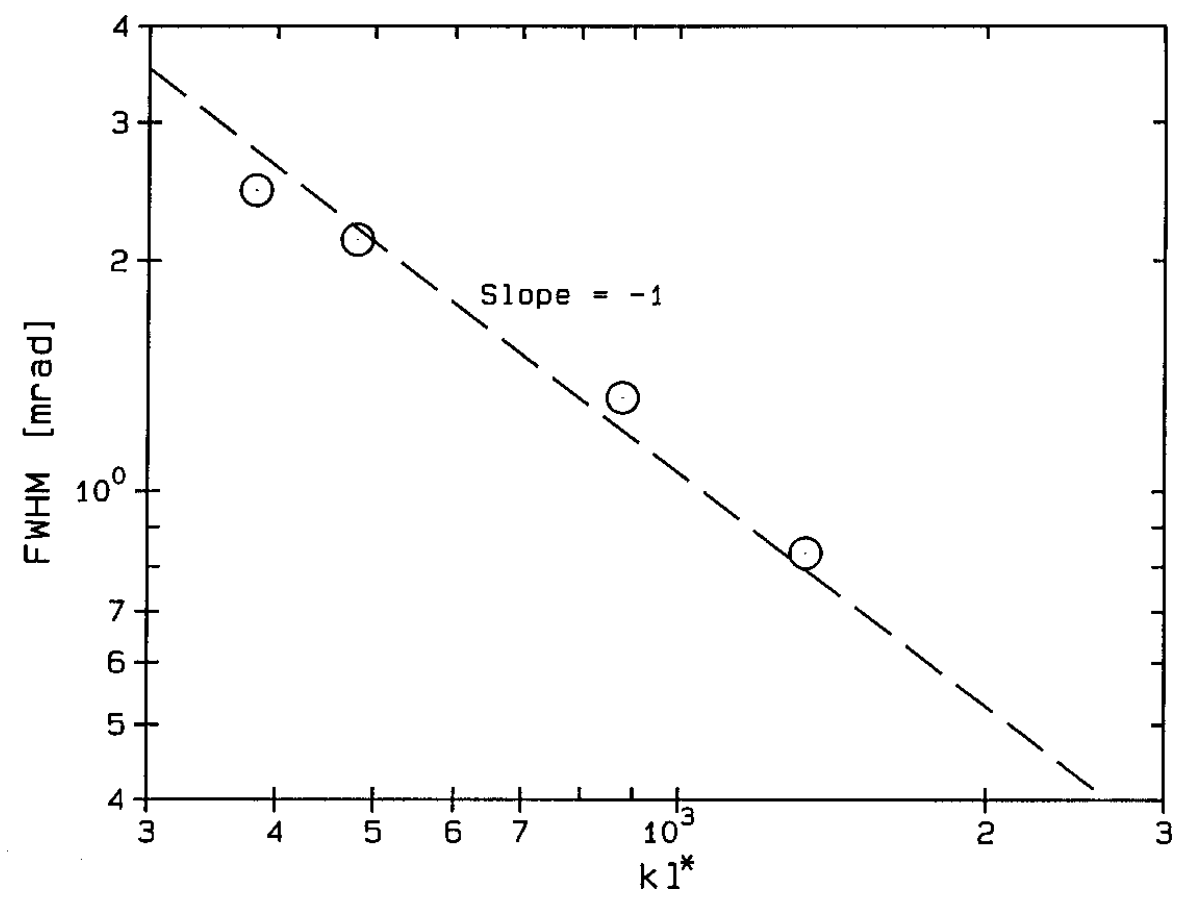

Figure 5: Coherent cone widths versus scaled transport mean-free-path length $k \ell^{*}$. The transport mean-free-path length $\ell^{*}$ was obtained from Mie theory appropriately corrected for particle-particle correlations. The full width at half maximum (FWHM) of these cones varies inversely with the scaled transport path length in agreement with theoretical prediction.

\section{CONCLUSIONS}

Coherent backscattering of light is an experiment that is ideally suited for an advanced undergraduate laboratory. It represents a topic of current interest within the research community and yet is readily accessible at the undergraduate level. Coherent backscattering of light is a phenomenon in which partial waves traversing time-reversed paths interfere constructively in the backscattering direction leading to the appearance of 
an intensity cone. It affords students an opportunity to learn to conduct a low light level experiment with precise angular resolution and to use sophisticated data analysis tools and techniques. Coherent backscattering intensity profiles have been obtained and the inverse dependence of their width on the transport mean-free-path length has been verified.

\section{Acknowledgments}

We are pleased to acknowledge support from a William and Flora Hewlett Foundation Award of Research Corporation and the donors of The Petroleum Research Fund, administered by the American Chemical Society, for support of this work. 


\section{REFERENCES}

[1] S. John, "Localization of Light," Physics Today 44 \#5, 32-40 (1991).

[2] Colloidal polystyrene suspensions may be obtained from Polysciences, Inc., Warrington, PA or Duke Scientific Corporation, Palo Alto, CA. Naturally occurring colloidal samples may also be used. We have obtained CBS intensity profiles from milk and baby formula.

[3] Y. Kuga, A. Ishimaru, "Retro reflection from a Dense Distribution of Theoretical Particles," J. Opt. Soc. Am. A1, 831-835 (1984).

[4] M. van Albada and A. Lagendijk, "Observation of Weak Localization of Light in a Random Medium,” Phys. Rev. Lett. 55, 2692-2695 (1985).

[5] G. Maret and P. Wolf, "Weak Localization and Coherent Backscattering of Photons in Disordered Media," Phys. Rev. Lett. 55, 2696-2699 (1985).

[6] R. L. Liboff, Kinetic Theory Classical, Quantum, and Relativistic Descriptions (Prentice Hall, Englewood Cliffs, NJ, 1990) pp. 180-181.

[7] P. Wolf, G. Maret, E. Akkermans, and R. Maynard, "Optical Coherent Backscattering by Random Media: an Experimental Study," J. Phys. Fr. 49, 63-75 (1988).

[8] M. van Albada, M. van der Mark, and A. Lagendijk, "Observation of Weak Localization of Light in a Finite Slab: Anisotropy Effects and Light-Path Classification," Phys. Rev. Lett. 58, 361-364 (1987).

[9] P. Saulnier and G. Watson, "Role of Surface Reflectivity in Coherent Backscattering Measurements," Optics Lett. 17, 1-3 (1992).

[10] W. Press, B. Flannery, S. Teukolsky, and W. Vetterling, Numerical Recipes in C (Cambridge University Press, New York, NY, 1990) pp. 517-565.

[11] C. Bohren and D. Huffman, Absorption and Scattering of Light by Small Particles (John Wiley \& Sons, New York, NY, 1983) pp. 82-129.

[12] P. Saulnier, M. Zinkin, and G. Watson, "Scatterer Correlation Effects on Photon Transport in Dense Random Media,” Phys. Rev. B 42, 2621-2623 (1990). 